\title{
Improving student understanding of corrections to the energy spectrum of the hydrogen atom for the Zeeman effect
}

\author{
Christof Keebaugh, Emily Marshman, and Chandralekha Singh \\ Department of Physics and Astronomy, University of Pittsburgh, Pittsburgh, Pennsylvania 15260, USA
}

(Received 18 July 2018; published 19 February 2019)

\begin{abstract}
We discuss an investigation of student difficulties with the corrections to the energy spectrum of the hydrogen atom for the intermediate field Zeeman effect using degenerate perturbation theory (DPT). The investigation was carried out in advanced quantum mechanics courses by administering free-response and multiple-choice questions and conducting individual interviews with students. We find that students share many common difficulties related to relevant physics concepts. They had difficulty with mathematical sense making in this context of quantum mechanics, which requires the ability to interpret the implications of the degeneracy in the unperturbed energy spectrum and how the Zeeman perturbation will impact the splitting of the energy levels. Many of the common student difficulties arise from challenges in mathematical sense making and applying linear algebra concepts incorrectly in this novel context of quantum mechanics. We describe how the research on student difficulties was used as a guide to develop and evaluate a Quantum Interactive Learning Tutorial (QuILT), which strives to help students develop a functional understanding of the concepts necessary for finding the corrections to the energy spectrum of the hydrogen atom for the intermediate field Zeeman effect using the DPT. We also discuss the development and validation of the DPT QuILT focusing on these issues and its in-class evaluation in the undergraduate and graduate courses.
\end{abstract}

DOI: 10.1103/PhysRevPhysEducRes.15.010113

\section{INTRODUCTION}

Quantum mechanics (QM) is challenging even for upperlevel undergraduate and graduate students, and students often struggle to make connections between mathematics and QM concepts in this abstract, nonintuitive, and novel context (e.g., see Refs. [1-22]). There have been a number of prior research studies aimed at investigating student reasoning in QM [23-34] and using the findings as resources for improving student understanding [35-43]. Guided by research studies conducted to identify student difficulties with QM and findings of cognitive research, we have been developing a set of research-based learning tools including the Quantum Interactive Learning Tutorials (QuILTs), which strive to help students develop a solid grasp of QM [44-56]. However, there has been relatively little research that focuses on student understanding of advanced topics in quantum mechanics, e.g., degenerate perturbation theory (DPT) [57-59]. The only prior work in this area has focused on investigating and improving student difficulties with fundamental concepts with DPT

Published by the American Physical Society under the terms of the Creative Commons Attribution 4.0 International license. Further distribution of this work must maintain attribution to the author(s) and the published article's title, journal citation, and DOI. in the context of a three-dimensional Hilbert space [59] and in the limiting cases of the strong and weak field Zeeman effects [58]. In the limiting cases of the strong and weak field Zeeman effect, one can use further the approximation method to determine the first order perturbative corrections and there is no need to explicitly diagonalize the perturbation matrix in each degenerate subspace of the unperturbed Hamiltonian. Here we discuss an investigation of student difficulties with mathematical sense making in a physical situation in the context of DPT involving the intermediate field Zeeman effect for the hydrogen atom. Unlike the case for the strong and weak field Zeeman effects, for the intermediate field Zeeman effect, one cannot use the limits to simplify the problem and one must explicitly diagonalize the perturbation $\hat{H}^{\prime}$ in each degenerate subspace of $\hat{H}^{0}$ before finding the perturbative corrections. This need for explicit diagonalization makes the intermediate field Zeeman effect problem technically more challenging. We also describe the development and validation of the research-based QuILT that uses student difficulties as resources and strives to help students learn to apply mathematical concepts in linear algebra correctly to find the corrections to the energy spectrum of the hydrogen atom for the Zeeman effect.

Prior research suggests that students often have difficulty applying mathematical concepts in the context of a concrete physical problem. In particular, students have difficulty 
connecting and applying mathematics correctly in physics contexts (e.g., see Refs. [60-64]). Mathematical sense making in the context of solving physics problems can often be more difficult than when solving equivalent mathematics problems without the physics context [60-64]. Since working memory is constrained to a limited number of chunks and students' knowledge chunks pertaining to a concept are small when they are learning and developing expertise in physics, use of mathematics in physics can increase the cognitive load during problem solving, especially if students are not proficient in mathematics [65], and students may struggle to integrate mathematical and physical concepts. Thus, sense making while focusing on solving a physics problem is often challenging and students sometimes make mathematical mistakes that they otherwise would not make if the physics context was absent [60-64].

One QM concept that involves mathematical sense making in a physical situation is degenerate perturbation theory (DPT) in the context of the Zeeman effect for the hydrogen atom. We investigated student difficulties with finding the first-order corrections to the energies of the hydrogen atom for the Zeeman effect using DPT and used the research as a guide to develop learning tools to improve student understanding.

The hydrogen atom has played a significant role in the development of quantum mechanics. Specifically, the discrete energy levels observed by spectroscopists for the hydrogen atom led Bohr to propose his model with quantized energy levels. Schrödinger proposed the wave model of particles involving the Schrödinger equation which explains features of the hydrogen atom well. The fine structure of the hydrogen atom is the combined effect of the relativistic correction and the spin-orbit interaction since the two components produce the same order of magnitude corrections to the energies compared to the unperturbed energies of the hydrogen atom. These fine structure corrections to the energies are smaller by a factor of $\alpha \approx 1 / 137$ squared, where $\alpha$ is the fine structure constant. The Zeeman effect represents the shift in the energy spectrum of the hydrogen atom due to the presence of a magnetic field. The shift in the energy spectrum due to the Zeeman effect is proportional to the strength of the magnetic field. The intermediate field Zeeman effect, which we will call the general case of the Zeeman effect (since it is more general than the strong or weak field Zeeman effect), is the focus here. It is the case in which the corrections to the energy spectrum due to the fine structure and Zeeman terms are comparable. While the Bohr model accurately explained the observed unperturbed energy levels of the hydrogen atom, it cannot explain or describe the observed energy shifts due to fine structure and Zeeman terms. Only the quantum mechanical treatment using the Schrödinger equation explains that the observed shifts are due to the fine structure and Zeeman terms. Spectroscopists can also identify the energy spectrum of the hydrogen atom under different conditions, e.g., in an external magnetic or electric field. Generally speaking, the interactions of the hydrogen atom with the external magnetic or electric field create shifts and splitting in the energy spectrum.

Here, we focus on the approximate solutions to the timeindependent Schrödinger equation (TISE) for the intermediate field Zeeman effect using perturbation theory.

While the solution for the TISE for the hydrogen atom with Coulomb potential energy can be solved exactly, the TISE for the hydrogen atom involving the Zeeman effect must include the fine structure correction term and cannot be solved exactly. The solution for the TISE for the hydrogen atom with Coulomb potential energy gives the unperturbed energies $E_{n}^{0}=-13.6 \mathrm{eV} / n^{2}$, where $n$ is the principal quantum number. Since the fine-structure term and, in general, the Zeeman term are significantly smaller than the unperturbed term in the Hamiltonian, perturbation theory is an excellent method for computing the corrections to the energies and comparing the theoretical results with experiments. The high degree of symmetry of the potential energy of the unperturbed Hamiltonian for the hydrogen atom leads to degeneracy in the energy spectrum of the hydrogen atom and DPT must be used to find the perturbative corrections for the Zeeman effect.

\section{BACKGROUND}

Below, we discuss the basics of DPT with which many students struggled and the development and validation of the QuILT, which strives to help students learn about DPT in the context of the Zeeman effect. Via the QuILT, students are provided guidance and support to determine a good basis for finding the perturbative corrections to the energies for the Zeeman effect, which includes corrections due to both the fine structure and Zeeman terms, and to calculate the perturbative corrections using that basis. For a given $\hat{H}^{0}$ and $\hat{H}^{\prime}$, a good basis consists of a complete set of eigenstates of $\hat{H}^{0}$ that diagonalizes $\hat{H}^{\prime}$ in each degenerate subspace of $\hat{H}^{0}$ [66].

\section{A. Basics for DPT}

Perturbation theory is a useful approximation method for finding the energies and the energy eigenstates for a system for which the TISE is not exactly solvable. The Hamiltonian $\hat{H}$ for the system can be expressed as the sum of two terms, the unperturbed Hamiltonian $\hat{H}^{0}$ and the perturbation $\hat{H}^{\prime}$, i.e., $\hat{H}=\hat{H}^{0}+\hat{H}^{\prime}$. The TISE for the unperturbed Hamiltonian, $\hat{H}^{0} \psi_{n}^{0}=E_{n}^{0} \psi_{n}^{0}$, (where $\psi_{n}^{0}$ is the $n$th unperturbed energy eigenstate and $E_{n}^{0}$ is the $n$th unperturbed energy), is exactly solvable. The energies can be approximated as $E_{n}=E_{n}^{0}+E_{n}^{1}+E_{n}^{2}+\ldots$ where $E_{n}^{i}$ for $i=1,2,3$.. are the $i$ th order corrections to the $n$th energy of the system. In PT, the first-order correction to the $n$th energy is 


$$
E_{n}^{1}=\left\langle\psi_{n}^{0}\left|\hat{H}^{\prime}\right| \psi_{n}^{0}\right\rangle
$$

and the first-order correction to the $n$th unperturbed energy eigenstate is

$$
\left|\psi_{n}^{1}\right\rangle=\sum_{m \neq n} \frac{\left\langle\psi_{m}^{0}\left|\hat{H}^{\prime}\right| \psi_{n}^{0}\right\rangle}{E_{n}^{0}-E_{m}^{0}}\left|\psi_{m}^{0}\right\rangle,
$$

in which $\left\{\left|\psi_{n}^{0}\right\rangle\right\}$ is a complete set of eigenstates of the unperturbed Hamiltonian $\hat{H}^{0}$. If the eigenvalue spectrum of $\hat{H}^{0}$ has degeneracy, the corrections to the energies and energy eigenstates are only valid provided one uses a good basis.

\section{B. Background for DPT involving the Zeeman effect}

For a hydrogen atom in an external magnetic field, one can use the DPT to find the corrections to the energy spectrum. Using standard notations, the unperturbed Hamiltonian $\hat{H}^{0}$ of a hydrogen atom is

$$
\hat{H}^{0}=\frac{\hat{p}^{2}}{2 m}-\frac{e^{2}}{4 \pi \epsilon_{0}} \frac{1}{r},
$$

which accounts only for the interaction of the electron with the nucleus via Coulomb attraction. The solution for the TISE for the hydrogen atom with Coulomb potential energy gives the unperturbed energies $E_{n}^{0}=-13.6 \mathrm{eV} / n^{2}$, where $n$ is the principal quantum number. The perturbation is

$$
\hat{H}^{\prime}=\hat{H}_{\mathrm{fs}}^{\prime}+\hat{H}_{Z}^{\prime},
$$

in which $\hat{H}_{Z}^{\prime}$ is the Zeeman term and $\hat{H}_{\mathrm{fs}}^{\prime}$ is the fine structure term. The Zeeman term accounts for the potential energy of the magnetic moments due to the orbital and spin angular momenta in the external magnetic field. The Zeeman term is given by

$$
\hat{H}_{Z}^{\prime}=\frac{\mu_{B} B_{\mathrm{ext}}}{\hbar}\left(\hat{L}_{z}+2 \hat{S}_{z}\right),
$$

in which $\vec{B}_{\text {ext }}=B_{\text {ext }} \hat{z}$ is a uniform, time independent external magnetic field along the $\hat{z}$ direction, $\mu_{B}$ is the Bohr magneton, and $\hat{L}_{z}$ and $\hat{S}_{z}$ are the operators corresponding to the $z$ component of the orbital and spin angular momenta, respectively. The fine structure term includes a relativistic correction for the kinetic energy and the spinorbit coupling, and is expressed as

$$
\hat{H}_{\mathrm{fs}}^{\prime}=\hat{H}_{r}^{\prime}+\hat{H}_{\mathrm{SO}}^{\prime} .
$$

Here,

$$
\hat{H}_{r}^{\prime}=-\frac{\hat{p}^{4}}{8 m^{3} c^{2}}
$$

is the relativistic correction term and

$$
\hat{H}_{\text {SO }}^{\prime}=\frac{e^{2}}{8 \pi \epsilon_{0}} \frac{1}{m^{2} c^{2} r^{3}} \vec{S} \cdot \vec{L}
$$

is the spin-orbit interaction term (all notations are standard).

We note that the unperturbed Hamiltonian is spherically symmetric since $\left[\hat{H}^{0}, \hat{\vec{L}}\right]=0$. Therefore, for a fixed $n, \hat{H}^{0}$ for the hydrogen atom is diagonal when any complete set of orthogonal states is chosen for the angular part of the basis (consisting of the product states of orbital and spin angular momenta). Thus, so long as the radial part of the basis is always chosen to be a stationary state wave function $R_{n l}(r)$ for the unperturbed hydrogen atom (for a given principal quantum number $n$ and the orbital angular momentum quantum number $l$ ), which we will assume throughout, the choice of a good basis amounts to choosing the angular part of the basis appropriately, i.e., ensuring that the perturbation is diagonal in each degenerate subspace of $\hat{H}^{0}$. Therefore, we focus on the angular part of the basis (or angular basis) to find a good basis and the corrections to the energies for the perturbation $\hat{H}^{\prime}$ corresponding to the intermediate field Zeeman effect in the hydrogen atom. For the angular basis for each $n$, states in the "coupled" representation $\left|n, l, j, m_{j}\right\rangle$ are labeled by the quantum numbers $l, s, j$, and $m_{j}$ (in addition to $n$ ) and the total angular momentum is defined as $\vec{J}=\vec{L}+\vec{S}$ (all notations are standard and $s$ has been suppressed from the states $\left|n, l, j, m_{j}\right\rangle$ since $s=1 / 2$ for the electron is a fixed value for a hydrogen atom). States in the coupled representation are eigenstates of $\hat{L}^{2}, \hat{S}^{2}, \hat{J}^{2}$, and $\hat{J}_{Z}$. On the other hand, for each $n$, states in the "uncoupled" representation $\left|n, l, m_{l}, m_{s}\right\rangle$ are labeled by the quantum numbers $l, m_{l}$, and $m_{s}$ (in addition to $n$ ), in which all notations are standard. States in the uncoupled representation are eigenstates of $\hat{L}^{2}, \hat{S}^{2}, \hat{L}_{Z}$, and $\hat{S}_{Z}$.

An angular basis consisting of states in the coupled representation forms a good basis for the fine structure term $\hat{H}_{\mathrm{fs}}^{\prime}$ since with this choice of the angular basis, $\hat{H}_{\mathrm{fs}}^{\prime}$ is diagonal in each degenerate subspace of $\hat{H}^{0}$. On the other hand, a basis consisting of states in the uncoupled representation forms a good angular basis for the Zeeman perturbation $\hat{H}_{Z}^{\prime}$ (in this case, first order PT yields the exact result since $\left.\left[\hat{H}^{0}, \hat{H}_{Z}^{\prime}\right]=0\right)$. Therefore, for the intermediate field Zeeman effect, in which $\hat{H}^{\prime}=\hat{H}_{\mathrm{fs}}^{\prime}+\hat{H}_{Z}^{\prime}$ and $\hat{H}_{\mathrm{fs}}^{\prime}$ and $\hat{H}_{Z}^{\prime}$ are treated on equal footing (we will use the notation $\hat{H}_{\mathrm{fs}}^{\prime} \approx \hat{H}_{Z}^{\prime}$ to denote that the energy corrections corresponding to $\hat{H}_{\mathrm{fs}}^{\prime}$ are comparable to $\hat{H}_{Z}^{\prime}$ ), neither a basis consisting of states in the coupled representation nor a basis consisting of states in the uncoupled representation forms a good angular basis to find perturbative corrections for the hydrogen atom placed in an external magnetic field. The following procedure describes how to determine a good 
angular basis to find the first order corrections to the energy spectrum for the intermediate field Zeeman effect:

(i) choose an initial basis consisting of a complete set of eigenstates of $\hat{H}^{0}$ (e.g., one is free to choose an angular basis consisting of states in the coupled representation or a basis consisting of states in the uncoupled representation or any other basis),

(ii) write the $\hat{H}^{0}$ and $\hat{H}^{\prime}$ matrices in the chosen basis,

(iii) identify $\hat{H}^{\prime}$ in each degenerate subspace of $\hat{H}^{0}$,

(iv) diagonalize the $\hat{H}^{\prime}$ matrix in each degenerate subspace of $\hat{H}^{0}$ to determine a good basis, and

(v) recognize that the first-order corrections to the energy spectrum are the diagonal matrix elements of the $\hat{H}^{\prime}$ matrix as given by Eq. (1) in the good basis.

\section{METHODOLOGY FOR INVESTIGATING STUDENT DIFFICULTIES}

Student difficulties with the corrections to the energies of the hydrogen atom for the Zeeman effect using DPT were investigated using five years of data involving responses from 64 upper-level undergraduate students and 42 firstyear graduate students at the University of Pittsburgh to open-ended and multiple-choice questions administered after traditional instruction in relevant concepts. The undergraduates were in an upper-level undergraduate QM course, and graduate students were in a graduate-level QM course. Additional insight was gained concerning these difficulties via responses of 13 students during a total of $45 \mathrm{~h}$ of individual interviews using the "think aloud" protocol in which they were asked to answer the questions aloud that were posed without being disturbed [67]. Only at the end, they were asked to clarify any issues. Students were provided with all relevant information discussed in the introduction and background section and had lecture-based instruction in relevant concepts. Similar percentages of undergraduate and graduate students displayed difficulties with DPT.

We first analyzed responses of 32 undergraduates on questions related to DPT in the context of the Zeeman effect for hydrogen atom administered in the two previous years.

Then, we examined the difficulties that 32 undergraduate and 42 graduate students had with identifying a good basis for the Zeeman effect in the following three years as part of an in-class quiz after traditional lecture-based instruction. In all questions, students were told that the radial part of the basis is chosen to be the stationary state wave function $R_{n l}(r)$. The following question is representative of a series of questions that were posed after traditional lecture-based instruction on relevant concepts and after students had engaged with the QuILT (the operator $\hat{H}^{\prime}$, in Q1, is a proxy for the operators $\hat{H}_{r}^{\prime}, \hat{H}_{\mathrm{SO}}^{\prime}, \hat{H}_{\mathrm{fs}}^{\prime}, \hat{H}_{Z}^{\prime}$, and $\hat{H}_{\mathrm{fs}}^{\prime}+\hat{H}_{Z}^{\prime}$ that were listed individually in five separate questions on the pretest after traditional, lecture-based instruction and post-test after engaging with the QuILT):
Q1. A perturbation $\hat{H}^{\prime}$ acts on a hydrogen atom with the unperturbed Hamiltonian $\hat{H}^{0}=-\frac{\hbar^{2}}{2 m} \nabla^{2}-\frac{e^{2}}{4 \pi \epsilon_{0}}\left(\frac{1}{r}\right)$. For the Hamiltonian $\hat{H}$, circle $\boldsymbol{A L L}$ of the representations that can be chosen as the angular part of a "good" basis and explain your reasoning. Assume that for all cases, the principal quantum number is restricted to $n=2$.

(i) Coupled representation,

(ii) Uncoupled representation,

(iii) Any arbitrary complete orthonormal basis constructed with linear combinations of states in the coupled representation with the same $l$ (i.e., states with different $l$ values are not mixed),

(iv) Any arbitrary complete orthonormal basis constructed with linear combinations of states in the uncoupled representation with the same $l$ (i.e., states with different $l$ values are not mixed),

(v) Neither coupled nor uncoupled representation.

Options iii and iv were administered to probe whether students realize that a good basis for a spherically symmetric operator $\left(\hat{H}_{r}^{\prime}\right)$ consists of any arbitrary complete orthonormal basis with the same $l$. Additionally, options iii and iv were designed to probe whether students realize that if the coupled representation forms a good basis, then it is not generally the case that any arbitrary complete orthonormal basis constructed with linear combinations of states in the coupled representation also forms a good basis. We note that options iii and iv were given without the condition of the same $l$ in one year of the study and that there was no difference in student performance based upon whether the wording of the question included the same $l$ or not for $\hat{H}_{\mathrm{SO}}^{\prime}$ and $\hat{H}_{\mathrm{fs}}^{\prime}$.

In order to find the first-order corrections to the energies, one must first choose a good basis. Q1 focuses on the bases that form a good basis for the perturbation Hamiltonian for the intermediate field Zeeman effect with $\hat{H}^{\prime}=\hat{H}_{\mathrm{fs}}^{\prime}+$ $\hat{H}_{Z}^{\prime}$, as well as the operators $\hat{H}_{\mathrm{fs}}^{\prime}$ and $\hat{H}_{Z}^{\prime}$ individually. Knowledge of the bases that form a good angular basis for the individual perturbation operators $\hat{H}_{\mathrm{fs}}^{\prime}$ and $\hat{H}_{Z}^{\prime}$ can be helpful when determining a good basis for the intermediate field Zeeman effect with the perturbation $\hat{H}^{\prime}=\hat{H}_{\mathrm{fs}}^{\prime}+\hat{H}_{Z}^{\prime}$.

The unperturbed Hamiltonian $\hat{H}^{0}$ is spherically symmetric with unperturbed energies only dependent on $n$ and therefore options i, ii, iii, and iv in Q1 all form a complete set of angular parts of the eigenstates of $\hat{H}^{0}$. Therefore, one must consider which set of angular basis states in Q1 also diagonalizes the given $\hat{H}^{\prime}$ in the degenerate subspace of $\hat{H}^{0}$. Since the given degenerate subspace of $\hat{H}^{0}$ corresponds to $n=2$, a good angular basis is one in which the perturbation matrix is also diagonal in that subspace.

In each degenerate subspace of $\hat{H}^{0}$, the fine structure term $\hat{H}_{\mathrm{fs}}^{\prime}$ is diagonal if the basis is chosen to consist of states in the coupled representation (option i in Q1) and the Zeeman term is diagonal if the basis is chosen to consist of 
states in the uncoupled representation (option ii in Q1), but not vice versa. Therefore, for the intermediate field Zeeman effect, in which the perturbation is $\hat{H}^{\prime}=\hat{H}_{\mathrm{fs}}^{\prime}+\hat{H}_{Z}^{\prime}$, neither a basis consisting of states in the coupled representation nor a basis consisting of states in the uncoupled representation forms a good basis and option v in Q1 is the correct answer. In order to determine a good basis for the intermediate field Zeeman effect, one may first choose an initial basis consisting of states in either the coupled or uncoupled representation and then diagonalize the perturbation $\hat{H}^{\prime}=$ $\hat{H}_{\mathrm{fs}}^{\prime}+\hat{H}_{Z}^{\prime}$ in the $n=2$ degenerate subspace of $\hat{H}^{0}$. Thus, students must first express either the $\hat{H}_{\mathrm{fs}}^{\prime}$ or $\hat{H}_{Z}^{\prime}$ matrix in an initial basis in which it is not diagonal in the degenerate subspace of $\hat{H}^{0}$. Then, they must be able to diagonalize the perturbation $\hat{H}^{\prime}=\hat{H}_{\mathrm{fs}}^{\prime}+\hat{H}_{Z}^{\prime}$ in the degenerate $n=2$ subspace of $\hat{H}^{0}$ and be able to find the corrections to the energy spectrum.

Below, we discuss some common difficulties with corrections to the energy spectrum of the hydrogen atom for the Zeeman effect found via research that interfere with students choosing a good basis and using DPT correctly in this context. We then discuss how the difficulties were used as a guide in the DPT QuILT to help students find the corrections to the energy spectrum due to the intermediate field Zeeman effect.

\section{STUDENT DIFFICULTIES}

Students had some difficulties with DPT in general (not restricted to the context of the Zeeman effect only). For example, when students were asked to determine a good basis for finding the corrections to the energies of the hydrogen atom, many students did not even realize that DPT should be used. Other students knew that they had to use DPT to find the corrections to the wavefunction, but they did not use DPT to find the first-order corrections to the energies. These students often incorrectly claimed that they did not need to use DPT since no terms in $E_{n}^{1}=$ $\left\langle\psi_{n}^{0}\left|\hat{H}^{\prime}\right| \psi_{n}^{0}\right\rangle$ "blow up."

In the context of the intermediate field Zeeman effect, some students only focused on the Zeeman term $\hat{H}_{Z}^{\prime}$ when asked to determine a good basis for finding the corrections to the energies of the hydrogen atom. In particular, they did not take into account the fine structure term $\hat{H}_{\mathrm{fs}}^{\prime}$ (omitted it altogether) and focused only on the Zeeman term as the perturbation. If the fine structure term $\hat{H}_{\mathrm{fs}}^{\prime}$ is neglected, then one can determine the exact energies for $\hat{H}^{0}+\hat{H}_{Z}^{\prime}$ and there is no need for perturbation theory since $\left[\hat{H}^{0}, \hat{H}_{Z}^{\prime}\right]=0$. However, the fine structure term should be considered when determining the corrections to the unperturbed energy spectrum.

As noted, to probe students' understanding of a good basis for the corrections to the energy spectrum due to the intermediate field Zeeman effect, students were asked question Q1. In the context of the intermediate field Zeeman effect, in which the perturbation is $\hat{H}^{\prime}=\hat{H}_{\mathrm{fs}}^{\prime}+$ $\hat{H}_{Z}^{\prime}$, students struggled to realize that neither a basis consisting of states in the coupled representation nor a basis consisting of states in the uncoupled representation forms a good basis for the perturbative corrections to the hydrogen atom placed in an external magnetic field. The results are summarized in Table I. We note that students were permitted to select more than one option in question Q1, and therefore, the sum of the percentages for all of the options on the pretest and posttest is greater than $100 \%$. Table I shows that only $44 \%$ of undergraduate students and 33\% of graduate students correctly identified that option $\mathrm{v}$ in $\mathrm{Q} 1$ is the correct answer for the Zeeman effect. Additionally, $16 \%$ of undergraduate and $17 \%$ of graduate students did not provide any answer to the multiple-choice question Q1 after traditional lecture-based instruction in relevant concepts.

Below, we discuss student difficulties that hinder their ability to select the representation that forms a good angular basis in Q1 and find the corrections to the energy spectrum. In this section, we focus on the qualitative results found primarily from student responses during the think-aloud interviews.

\section{A. Difficulty understanding why diagonalizing the entire $\hat{H}^{\prime}$ matrix is problematic when $\hat{H}^{0}$ and $\hat{H}^{\prime}$ do not commute}

More than half of the interviewed students (62\%) did not realize that when the initially chosen basis is not a good basis and the unperturbed Hamiltonian $\hat{H}^{0}$ and the perturbing Hamiltonian $\hat{H}^{\prime}=\hat{H}_{\mathrm{fs}}^{\prime}+\hat{H}_{Z}^{\prime}$ do not commute, they must diagonalize the $\hat{H}^{\prime}=\hat{H}_{\mathrm{fs}}^{\prime}+\hat{H}_{Z}^{\prime}$ matrix only in the degenerate subspace of $\hat{H}^{0}$. When presented with a similar system and asked to determine the first order corrections to the energies, one interviewed student who attempted to diagonalize the entire $\hat{H}^{\prime}$ matrix justified his reasoning by

TABLE I. The percentage of students who chose the listed options as representations to form a good basis for the perturbation $\hat{H}^{\prime}=\hat{H}_{\mathrm{fs}}^{\prime}+\hat{H}_{Z}^{\prime}$ and the unperturbed Hamiltonian $\hat{H}^{0}$ and the percentage of students who did not select any option in Q1 on the pretest and post-test for undergraduate students $(N=32)$ and graduate students $(N=42)$. The correct answer (option v) is in boldface text.

\begin{tabular}{lccccc}
\hline \hline & \multicolumn{2}{c}{ Undergraduate students } & & \multicolumn{2}{c}{ Graduate students } \\
\cline { 2 - 3 } \cline { 5 - 6 } Option & Pretest $(\%)$ & Post-test $(\%)$ & & Pretest $(\%)$ & Post-test $(\%)$ \\
\hline i & 28 & 3 & & 29 & 17 \\
ii & 22 & 0 & & 17 & 17 \\
iii & 16 & 3 & & 12 & 10 \\
iv & 13 & 0 & & 12 & 10 \\
v & $\mathbf{4 4}$ & $\mathbf{9 7}$ & & $\mathbf{3 3}$ & $\mathbf{8 3}$ \\
Blank & 16 & 0 & & 17 & 0 \\
\hline \hline
\end{tabular}


incorrectly stating, "We must find the simultaneous eigenstates of $\hat{H}^{0}$ and $\hat{H}^{\prime}$." This student, and many others, did not realize that when $\hat{H}^{0}$ and $\hat{H}^{\prime}=\hat{H}_{\mathrm{fs}}^{\prime}+\hat{H}_{Z}^{\prime}$ do not commute, we cannot simultaneously diagonalize $\hat{H}^{0}$ and $\hat{H}^{\prime}=\hat{H}_{\mathrm{fs}}^{\prime}+$ $\hat{H}_{Z}^{\prime}$ since they do not share a complete set of eigenstates. Students struggled with the fact that if $\hat{H}^{0}$ and $\hat{H}^{\prime}=\hat{H}_{\mathrm{fs}}^{\prime}+$ $\hat{H}_{Z}^{\prime}$ do not commute, diagonalizing $\hat{H}^{\prime}=\hat{H}_{\mathrm{fs}}^{\prime}+\hat{H}_{Z}^{\prime}$ produces a basis in which $\hat{H}^{0}$ is not diagonal. Also, since $\hat{H}^{0}$ is the dominant term and $\hat{H}^{\prime}=\hat{H}_{\mathrm{fs}}^{\prime}+\hat{H}_{Z}^{\prime}$ provides only small corrections, we must ensure that the basis states used to determine the perturbative corrections in Eqs. (1) and (2) remain eigenstates of $\hat{H}^{0}$.

\section{B. Incorrectly claiming that both the coupled and uncoupled representations are good bases}

Many students had difficulty identifying a good basis for perturbative corrections for the intermediate field Zeeman effect. For example, in Q1, 22\% of undergraduates and $29 \%$ of the graduate students correctly identified that the good angular basis for the fine structure term $\hat{H}_{\mathrm{fs}}^{\prime}$ is a basis consisting of states in the coupled representation (option i) and also correctly identified that the good angular basis for the Zeeman term $\hat{H}_{Z}^{\prime}$ is a basis consisting of states in the uncoupled representation (option ii in Q1). However, after correctly identifying the good angular basis for the two perturbations individually, some of these students did not realize that neither the coupled nor the uncoupled representation (option $\mathrm{v}$ in Q1) forms a good angular basis for the Zeeman effect in which the perturbation is $\hat{H}^{\prime}=\hat{H}_{\mathrm{fs}}^{\prime}+\hat{H}_{Z}^{\prime}$. One interviewed student incorrectly claimed that "the coupled are a good basis for $\hat{H}_{\mathrm{fs}}^{\prime}$ and uncoupled are a good basis for $\hat{H}_{Z}^{\prime}$, so both coupled and uncoupled form a good basis for $\hat{H}_{\mathrm{fs}}^{\prime}+\hat{H}_{Z}^{\prime}$." This student and others with this type of response incorrectly thought that since a basis consisting of states in the coupled representation (option $\mathrm{i}$ in Q1) forms a good basis for the fine structure term $\hat{H}_{\mathrm{fs}}^{\prime}$ and a basis consisting of states in the uncoupled representation (option ii in Q1) forms a good angular basis for the Zeeman term $\hat{H}_{Z}^{\prime}$, a good basis for the perturbation consisting of the sum of these two perturbations is a basis consisting of states in either the coupled or uncoupled representation.

\section{Incorrectly claiming that a good basis does not exist for the Zeeman effect}

In Q1, some students who correctly identified that the good angular basis for the fine structure term $\hat{H}_{\mathrm{fs}}^{\prime}$ is a basis consisting of states in the coupled representation and also correctly identified that a good angular basis for the Zeeman term $\hat{H}_{Z}^{\prime}$ is a basis consisting of states in the uncoupled representation correctly chose that neither the coupled nor the uncoupled representation forms a good basis for the perturbation $\hat{H}^{\prime}=\hat{H}_{\mathrm{fs}}^{\prime}+\hat{H}_{Z}^{\prime}$ (option $\mathrm{v}$ in Q1) but then used incorrect reasoning to do so. Two common examples are as follows:

Some students incorrectly argued that since neither an angular basis consisting of states in the coupled representation nor a basis consisting of states in the uncoupled representation forms a good basis, a good basis does not exist for this case. They struggled to realize that the coupled representation or the uncoupled representation were not the only two possibilities for the angular part of the basis. One interviewed student with this type of reasoning had difficulty understanding the meaning of options iii and iv in Q1, stating: "I don't know what a linear combination of coupled or uncoupled states is. I thought there were just coupled states or uncoupled states." This student and others with this type of reasoning did not realize that a good basis could be constructed from a linear combination of states in the coupled representation (or equivalently a linear combination of states in the uncoupled representation).

Some students had difficulty realizing that any linear combination of states from the same degenerate subspace of $\hat{H}^{0}$ are also eigenstates of $\hat{H}^{0}$. For example, one student who correctly identified that neither the coupled nor the uncoupled representation forms a good basis for the Zeeman effect argued that "no good basis exists since we cannot diagonalize a part of the $\hat{H}^{\prime}$ matrix $\left(\hat{H}^{\prime}\right.$ matrix in the degenerate subspace of $\hat{H}^{0}$ ) without affecting the $\hat{H}^{0}$ matrix." This student and others who provided similar incorrect reasoning claimed that by diagonalizing $\hat{H}^{\prime}$ in the degenerate subspace of $\hat{H}^{0}$, the $\hat{H}^{0}$ matrix would no longer be diagonal. However, due to the degeneracy, ANY linear combination of states from the same degenerate subspace of $\hat{H}^{0}$ are eigenstates of $\hat{H}^{0}$. Therefore, diagonalizing $\hat{H}^{\prime}$ in the degenerate subspace of $\hat{H}^{0}$ determines the special linear combination that forms a good basis.

\section{Incorrectly claiming that the choice of the initial basis affects corrections to the energy spectrum}

Of the students who correctly identified that a good basis for the Zeeman effect will consist of a special linear combination of states in the coupled representation (or, equivalently, a special linear combination of states in the uncoupled representation), many did not realize that the first order corrections to the energy spectrum would be the same regardless of the initial choice of the basis. Since neither a basis consisting of states in the coupled representation nor a basis consisting of states in the uncoupled representation forms a good basis, a good basis cannot easily be identified at the onset. In order to determine a good basis and the first order corrections to the energy spectrum due to the Zeeman effect, one can initially choose a basis consisting of states in the coupled representation and then diagonalize $\hat{H}^{\prime}=\hat{H}_{\mathrm{fs}}^{\prime}+\hat{H}_{Z}^{\prime}$ in each degenerate subspace of $\hat{H}^{0}$. However, one could also initially choose a 
basis consisting of states in the uncoupled representation and then diagonalize $\hat{H}^{\prime}=\hat{H}_{\mathrm{fs}}^{\prime}+\hat{H}_{Z}^{\prime}$ in each degenerate subspace of $\hat{H}^{0}$ to determine a good basis and the first order corrections to the energy spectrum due to the Zeeman effect. Regardless of the choice of the initial basis, after diagonalizing $\hat{H}^{\prime}=\hat{H}_{\mathrm{fs}}^{\prime}+\hat{H}_{Z}^{\prime}$ in each degenerate subspace of $\hat{H}^{0}$, the first order corrections to the energy spectrum due to the Zeeman effect will be the same in any good basis. Many students thought that the first order corrections to the energies depended on the initial choice of basis. Therefore, if one chooses a basis consisting of states in the coupled representation then the first order corrections in this case would be different than those obtained had a basis consisting of states in the uncoupled representation been chosen as the initial basis. However, it does not make sense experimentally that the observed perturbative corrections would depend upon the choice of basis. Lack of appropriate connection between physics and mathematics in the context of DPT for the Zeeman effect sheds light on the difficulty students have in mathematical sense making in QM. It also sheds light on the physics epistemology pertaining to whether one should get the same perturbative corrections in experiments regardless of the choice of initial basis or whether the initial choice of basis should impact what is experimentally measured values of energies.

\section{E. Making computational mistakes while attempting to diagonalize the entire eight-dimensional $\hat{H}^{\prime}$ matrix instead of diagonalizing the two separate $2 \times 2$ submatrices of the block diagonal matrix $\hat{\boldsymbol{H}}^{\prime}=\hat{\boldsymbol{H}}_{\mathrm{fs}}^{\prime}+\hat{\boldsymbol{H}}_{Z}^{\prime}$}

When asked to determine the first order corrections to the energies for the intermediate field Zeeman effect for the $n=2$ degenerate subspace of $\hat{H}^{0}$, some students correctly identified that one can initially choose either a basis consisting of states in the coupled representation or a basis consisting of states in the uncoupled representation and then diagonalize $\hat{H}^{\prime}=\hat{H}_{\mathrm{fs}}^{\prime}+\hat{H}_{Z}^{\prime}$ in each degenerate subspace of $\hat{H}^{0}$. For example, in a basis consisting of states in the coupled representation $\left(\left|n, l, j m_{j}\right\rangle\right)$, the perturbation matrix $\hat{H}^{\prime}=\hat{H}_{Z}^{\prime}+\hat{H}_{\mathrm{fs}}^{\prime}$ corresponding to the $n=2$ subspace is given below [in which $\gamma=(\alpha / 8)^{2} 13.6 \mathrm{eV}$, $\alpha=e^{2} / 4 \pi \epsilon_{0} \hbar c, \beta=\mu_{B} B_{\text {ext }}$, and the basis states are chosen in the order $\left|2,0, \frac{1}{2}, \frac{1}{2}\right\rangle,\left|2,0, \frac{1}{2},-\frac{1}{2}\right\rangle,\left|2,1, \frac{3}{2}, \frac{3}{2}\right\rangle,\left|2,1, \frac{3}{2},-\frac{3}{2}\right\rangle$, $\left|2,1, \frac{3}{2}, \frac{1}{2}\right\rangle,\left|2,1, \frac{1}{2}, \frac{1}{2}\right\rangle,\left|2,1, \frac{3}{2},-\frac{1}{2}\right\rangle$, and $\left.\left|2,1, \frac{1}{2},-\frac{1}{2}\right\rangle\right]$ :

$$
\hat{H}^{\prime}=-\left[\begin{array}{cccccccc}
5 \gamma-\beta & 0 & 0 & 0 & 0 & 0 & 0 & 0 \\
0 & 5 \gamma+\beta & 0 & 0 & 0 & 0 & 0 & 0 \\
0 & 0 & \gamma-2 \beta & 0 & 0 & 0 & 0 & 0 \\
0 & 0 & 0 & \gamma+2 \beta & 0 & 0 & 0 & 0 \\
0 & 0 & 0 & 0 & \gamma-\frac{2}{3} \beta & \frac{\sqrt{2}}{3} \beta & 0 & 0 \\
0 & 0 & 0 & 0 & \frac{\sqrt{2}}{3} \beta & 5 \gamma-\frac{1}{3} \beta & 0 & 0 \\
0 & 0 & 0 & 0 & 0 & 0 & \gamma+\frac{2}{3} \beta & \frac{\sqrt{2}}{3} \beta \\
0 & 0 & 0 & 0 & 0 & 0 & \frac{\sqrt{2}}{3} \beta & 5 \gamma+\frac{1}{3} \beta
\end{array}\right]
$$

However, when finding the corrections to the energy spectrum, some students attempted to diagonalize the entire $8 \times 8 \hat{H}^{\prime}$ matrix in the $n=2$ degenerate subspace of $\hat{H}^{0}$. While this approach is correct, it is easier to diagonalize the $8 \times 8 \hat{H}^{\prime}$ matrix by diagonalizing $\hat{H}^{\prime}$ only in the block diagonal subspaces with smaller dimensions than the initial $8 \times 8 \hat{H}^{\prime}$ matrix, i.e., the two separate $2 \times 2$ matrices

$$
-\left[\begin{array}{cc}
\gamma-\frac{2}{3} \beta & \frac{\sqrt{2}}{3} \beta \\
\frac{\sqrt{2}}{3} \beta & 5 \gamma-\frac{1}{3} \beta
\end{array}\right]
$$

and

$$
-\left[\begin{array}{cc}
\gamma+\frac{2}{3} \beta & \frac{\sqrt{2}}{3} \beta \\
\frac{\sqrt{2}}{3} \beta & 5 \gamma+\frac{1}{3} \beta
\end{array}\right] .
$$

In general, an expertlike approach to diagonalizing the $\hat{H}^{\prime}$ matrix involves diagonalizing $\hat{H}^{\prime}$ in the block diagonal subspaces with smaller dimensions and mathematical mistakes are less likely using this approach. However, many students did not realize that in order to determine a good basis, one can diagonalize these block diagonal subspaces in order to diagonalize the entire $\hat{H}^{\prime}$ matrix in the degenerate subspace of $\hat{H}^{0}$. In other words, they struggled with the fact that to diagonalize $\hat{H}^{\prime}$ in the $n=$ 2 degenerate subspace of $\hat{H}^{0}$, one can diagonalize the two separate $2 \times 2$ matrices instead of diagonalizing the entire 
$\hat{H}^{\prime}$ matrix in the $n=2$ subspace and obtain the linear combination of the states in the coupled representation that forms a good basis for finding the perturbative corrections for the Zeeman effect.

\section{METHODOLOGY FOR DEVELOPMENT OF THE QuILT}

\section{A. Development and validation of the QuILT}

The difficulties described show that many students struggle in determining a good basis for finding the corrections to the energy spectrum for the Zeeman effect. Therefore, we developed a QuILT [68] that takes into account these difficulties and strives to help students build a robust knowledge structure of these concepts. The development of the DPT QuILT started with an investigation of student difficulties via open-ended and multiple-choice questions administered after traditional instruction to advanced undergraduate and graduate students and conducting a cognitive task analysis from an expert perspective of the requisite knowledge [69]. The QuILT strives to help students build on their prior knowledge and addresses common difficulties found via research, some of which were discussed in the previous section.

The QuILT is inspired by Piaget's "optimal mismatch" framework as well as the preparation for future learning framework of Bransford and Schwartz. In Piaget's optimal mismatch framework, students are intentionally placed in a situation in which their current knowledge structures are inadequate and the students are required to reorganize existing structures or develop new structures to reconcile this conflict [70]. Bransford and Schwartz's preparation for future learning framework emphasizes that learning occurs when elements of innovation and efficiency are both present [71]. Innovation and efficiency describe two orthogonal components of instruction. Innovation describes aspects that are new to students, such as new concepts or new problem-solving skills. Efficiency is a measure of the structure and organization of the material, as well as how proficient the student is with the material. Instruction that incorporates only one of these elements leads to students becoming disengaged. If instruction is too innovative, students cannot connect the material with their prior knowledge and become frustrated. When the instruction is too efficient, students interact with repetitious material that does not provide intellectual stimulation and may become routine experts. However, they will not be able to transfer their learning to new situations.

In the QuILT, students are presented with innovative tasks. Whether it be examples, hypothetical conversations, or calculations, the QuILT strives to help students develop a deeper understanding by actively working through the inquiry-based learning sequences. Student difficulties are incorporated in these examples and conversations to create a cognitive conflict in which the students are then guided through additional tasks designed to resolve these issues. Efficiency is addressed in the QuILT in several ways. First, the QuILT follows the sequence laid out in the cognitive task analysis. It is organized in a manner that attempts to build on the students' prior knowledge, and each section in the QuILT builds upon the previous section. Second, students are provided scaffolding designed to help address common difficulties, thus reducing the cognitive conflict. Third, the QuILT progressively reduces the scaffolding to help students solve problems without any assistance. Finally, as the students work through the different tasks, they develop more proficiency at identifying the concepts and answering the questions.

The development of the QuILT went through a cyclic, iterative process. The preliminary version was developed based upon the cognitive task analysis and knowledge of common student difficulties. Next, the QuILT underwent many iterations among the three researchers and then was iterated several times with three physics faculty members to ensure that they agreed with the content and wording. It was also administered to graduate and advanced undergraduate students in individual think-aloud interviews to ensure that the guided approach was effective, the questions were unambiguously interpreted, and to better understand the rationale for student responses. During these semistructured interviews, students were asked to think aloud while answering the questions. Students first read the questions on their own and answered them without interruptions except that they were prompted to think aloud if they were quiet for a long time. After students had finished answering a particular question to the best of their ability, they were asked to further clarify and elaborate on issues that they had not clearly addressed earlier. The next step involved evaluating the impact of the QuILT on student learning and determining if the difficulties remained. Finally, modifications and improvements were made based upon the student and faculty feedback before it was administered to students in various QM courses.

\section{B. Structure of the QuILT}

The QuILT uses a guided inquiry-based approach to learning and actively engages students in the learning process. It includes a pretest to be administered in class after traditional instruction in DPT. Then, students engage with the tutorial in small groups in class (or alone when using it as a self-paced learning tool in homework), and finally a post-test is administered in class. As students work through the tutorial, they are asked to predict what should happen in a given situation. Then, the tutorial strives to provide scaffolding and feedback as needed to bridge the gap between their initial knowledge and the level of understanding that is desired. Students are also provided checkpoints to reflect upon what they have learned and make explicit connections between what they are learning and their prior knowledge. They are given an opportunity in 
the checkpoints to reconcile differences between their predictions and the guidance provided before proceeding further.

The DPT QuILT uses a blend of guided inquiry-based learning sequences involving both qualitative and quantitative reasoning to improve students' understanding. For example, the QuILT requires qualitative reasoning while students reason about hypothetical student conversations and quantitative reasoning to determine the matrix elements of the perturbations $\hat{H}_{\text {SO }}^{\prime}$ and $\hat{H}_{Z}^{\prime}$ in the coupled and uncoupled representations.

\section{Addressing student difficulties via guided learning sequences in the QuILT}

In the guided inquiry-based learning sequences in the QuILT, students actively engage with examples involving DPT in which they consider the perturbations $\hat{H}_{\mathrm{fs}}^{\prime}, \hat{H}_{Z}^{\prime}$, and $\hat{H}_{\mathrm{fs}}^{\prime}+\hat{H}_{Z}^{\prime}$ as the perturbation on $\hat{H}^{0}$. In this manner, students focus on the concepts involved in determining a good basis for the fine structure and Zeeman corrections to the energy spectrum of the hydrogen atom separately before considering $\hat{H}^{\prime}=\hat{H}_{\mathrm{fs}}^{\prime}+\hat{H}_{Z}^{\prime}$. For the unperturbed Hamiltonian $\hat{H}^{0}$ and the perturbation $\hat{H}^{\prime}=\hat{H}_{\mathrm{fs}}^{\prime}+\hat{H}_{Z}^{\prime}$, students learn about (i) why DPT must be used (ii) why care must be taken to choose a good basis for the Zeeman effect and (iii) how to find perturbative corrections to the energy spectrum. Below, we discuss how the QuILT strives to address student difficulties and help them learn about the perturbative corrections to the energy spectrum of the hydrogen atom due to the Zeeman effect using DPT.

Students first work through a warm-up for the tutorial that strives to help them identify the bases that consist of a complete set of eigenstates of operator $\hat{H}^{0}$ and the bases in which the operators $\hat{H}_{\mathrm{fs}}^{\prime}$ and $\hat{H}_{Z}^{\prime}$ are diagonal in each degenerate subspace of $\hat{H}^{0}$. In addition, students also work through examples in which they must determine the matrix elements of the operators $\hat{H}_{\text {SO }}^{\prime}$ or $\hat{H}_{Z}^{\prime}$. For example, they calculate several diagonal and off-diagonal matrix elements of $\hat{H}_{\mathrm{SO}}^{\prime}$ and $\hat{H}_{Z}^{\prime}$ in both a basis consisting of states in the coupled representation and a basis consisting of states in the uncoupled representation. Students were asked to focus on calculating the matrix elements of the operator $\hat{H}_{\text {SO }}^{\prime}$ in order to help them determine whether a basis consisting of states in the coupled or uncoupled representation forms a good basis for the fine structure perturbation $\hat{H}_{\mathrm{fs}}^{\prime}$. Since the fine structure term is $\hat{H}_{\mathrm{fs}}^{\prime}=\hat{H}_{r}^{\prime}+\hat{H}_{\mathrm{SO}}^{\prime}$, one must consider both $\hat{H}_{r}^{\prime}$ and $\hat{H}_{\mathrm{SO}}^{\prime}$ when determining a good basis. However, the relativistic term $\hat{H}_{r}^{\prime}$ is spherically symmetric with energy depending on $n$ and $l$ and so $\hat{H}_{r}^{\prime}$ is diagonal in each degenerate subspace of $\hat{H}^{0}$ for a basis consisting of states in the coupled or uncoupled representation for each fixed $n$ and $l$. Students were asked to focus on the angular part of the basis that makes $\hat{H}_{\text {SO }}^{\prime}$ diagonal in each degenerate subspace of $\hat{H}^{0}$. The warmup strives to help students learn the prerequisites for finding a good basis for the hydrogen atom for the Zeeman effect in the context of DPT.

Helping students identify a good basis for the fine structure term $\hat{H}_{\mathrm{fs}}^{\prime}$, the Zeeman term $\hat{H}_{Z}^{\prime}$, and $\hat{H}^{\prime}=$ $\hat{H}_{\mathrm{fs}}^{\prime}+\hat{H}_{Z}^{\prime}$ : The QuILT strives to help students learn that neither a basis consisting of states in the coupled representation nor a basis consisting of states in the uncoupled representation forms a good basis for the intermediate field Zeeman effect. As part of a guided inquiry-based sequence, students are asked to evaluate the validity of the following two statements in a hypothetical student conversation in the QuILT designed to scaffold students' learning:

Student 1: Since the coupled representation is a good basis for the fine structure term and the uncoupled representation is a good basis for the Zeeman term, both the coupled and uncoupled representation form good bases and are equally appropriate to find the first order corrections to the energies for $\hat{H}^{\prime}=\hat{H}_{\mathrm{fs}}^{\prime}+\hat{H}_{Z}^{\prime}$.

Student 2: I disagree with Student 1. You cannot consider different bases for different parts of $\hat{H}^{\prime}$. If we choose the coupled representation, $\hat{H}^{\prime}=\hat{H}_{\mathrm{fs}}^{\prime}+\hat{H}_{Z}^{\prime}$ is not diagonal in each degenerate subspace of $\hat{H}^{0}$ since $\hat{H}_{Z}^{\prime}$ is not diagonal in the coupled representation. Similarly, if we choose the uncoupled representation, $\hat{H}^{\prime}=\hat{H}_{\mathrm{fs}}^{\prime}+\hat{H}_{\mathrm{Z}}^{\prime}$, is not diagonal in each degenerate subspace of $\hat{H}^{0}$ since $\hat{H}_{\mathrm{fs}}^{\prime}$ is not diagonal in the uncoupled representation. Neither of these representations form a good basis.

Explain why you agree or disagree with Student 1 or Student 2.

Following this conversation, further scaffolding is provided through inquiry-based learning sequences which strive to help students reconcile that student 2 is correct.

Students are also given scaffolding support to help them determine a good basis and first-order corrections to the energy spectrum of the hydrogen atom for the Zeeman effect. The guided inquiry-based sequences in the QuILT strive to help students learn that neither a basis consisting of states in the coupled or uncoupled representation form a good basis. After diagonalizing $\hat{H}^{\prime}=\hat{H}_{\mathrm{fs}}^{\prime}+\hat{H}_{Z}^{\prime}$ in the $n=$ 2 degenerate subspace of $\hat{H}^{0}$, a good basis is obtained which consists of a linear combination of states in the coupled (or, equivalently, the uncoupled) representation. Students are provided checkpoints that allow them to reconcile any differences between their initial reasoning and the correct reasoning.

Helping students realize that the initial choice of basis cannot affect the corrections to the energy spectrum: The QuILT strives to help students learn that one is free to choose either an initial basis consisting of states in the 
coupled representation or a basis consisting of states in the uncoupled representation and then diagonalize the perturbation $\hat{H}^{\prime}=\hat{H}_{\mathrm{fs}}^{\prime}+\hat{H}_{Z}^{\prime}$ in each degenerate subspace of $\hat{H}^{0}$ in order to determine a good basis (and the first order corrections to the energies due to the Zeeman effect). The following statements from a hypothetical student conversation from a guided inquiry-based sequence in the QuILT strive to help students learn that the initial choice of basis cannot change the first order corrections to the energy spectrum due to the Zeeman effect once a good basis has been found.

Student 1: Since the diagonal matrix elements of $\hat{H}^{\prime}$ will depend on the choice of initial basis, a different choice of the initial basis in which we diagonalize $\hat{H}^{\prime}$ in the degenerate subspace of $\hat{H}^{0}$ will change the first order corrections to the energies.

Student 2: I disagree with Student 1. After diagonalizing $\hat{H}^{\prime}$ in each degenerate subspace of $\hat{H}^{0}$, a good basis is obtained and the first order correction to the energy will be the same regardless of which basis, e.g., the coupled or uncoupled representation, you had initially chosen. In a good basis, you will end up with the same diagonal matrix elements of $\hat{H}^{\prime}$ which are the first order corrections to the energies.

Explain why you agree or disagree with each student.

Students are provided additional scaffolding support to help them reconcile that student 2 is correct in the preceding conversation. In a good basis, the diagonal matrix elements of the perturbation $\hat{H}^{\prime}$ are the first order corrections to the energies regardless of the choice of the initial basis.

Helping students reflect upon the fact that diagonalizing the two separate $2 \times 2$ submatrices of the block diagonal matrix $\hat{H}^{\prime}=\hat{H}_{\mathrm{fs}}^{\prime}+\hat{H}_{Z}^{\prime}$ diagonalizes $\hat{H}^{\prime}$ in the $n=2$ subspace: In the QuILT, when basis states are chosen to be states in the coupled representation in an appropriate order, the $\hat{H}^{\prime}=\hat{H}_{\mathrm{fs}}^{\prime}+\hat{H}_{Z}^{\prime}$ matrix is block diagonal. Students are provided scaffolding support to help them realize that one is free to choose the initial angular basis states in any order to construct the matrices without affecting the first order corrections to the energy spectrum and that choosing basis states in a certain order may make determining the first order corrections to the energy spectrum easier to calculate. In particular, the QuILT strives to help students learn that in order to determine a good basis for the Zeeman effect in the $n=2$ subspace, one can diagonalize the block diagonal matrix $\hat{H}^{\prime}=\hat{H}_{\mathrm{fs}}^{\prime}+\hat{H}_{Z}^{\prime}$ by diagonalizing the two separate $2 \times 2$ submatrices of the block diagonal $\hat{H}^{\prime}=\hat{H}_{\mathrm{fs}}^{\prime}+\hat{H}_{Z}^{\prime}$ matrix rather than diagonalizing the entire $8 \times 8 \hat{H}^{\prime}=$ $\hat{H}_{\mathrm{fs}}^{\prime}+\hat{H}_{Z}^{\prime}$ matrix if the basis states are chosen in the order given earlier. The following student conversation regarding diagonalizing the $\hat{H}^{\prime}=\hat{H}_{\mathrm{fs}}^{\prime}+\hat{H}_{Z}^{\prime}$ matrix in the $n=2$ degenerate subspace of $\hat{H}^{0}$ for the Zeeman effect is part of a guided inquiry-based sequence in which students must reason about and explain whether each hypothetical student's statement is correct:

Student 1: In the case of $n=2, \hat{H}^{0}$ possesses an eightfold degeneracy, which means that in order to find a good basis for the correction to the $n=2$ energy spectrum, we must diagonalize the entire $8 \times 8 \hat{H}^{\prime}$ matrix in the $n=2$ degenerate subspace of $\hat{H}^{0}$.

Student 2: We must make an effort to diagonalize $\hat{H}^{\prime}$ only in those block diagonal subspaces with smaller dimensions in order to diagonalize the entire $\hat{H}^{\prime}$ matrix in the degenerate subspace of $\hat{H}^{0}$ to obtain the good basis set. When I calculate the $\hat{H}^{\prime}$ matrix for $n=2$ in the coupled representation and the angular basis states are chosen in the order $\left|\psi_{1}\right\rangle=\left|2,0, \frac{1}{2}, \frac{1}{2}\right\rangle, \quad\left|\psi_{2}\right\rangle=$ $\left|2,0, \frac{1}{2},-\frac{1}{2}\right\rangle, \quad\left|\psi_{3}\right\rangle=\left|2,1, \frac{3}{2}, \frac{3}{2}\right\rangle, \quad\left|\psi_{4}\right\rangle=\left|2,1, \frac{3}{2},-\frac{3}{2}\right\rangle$, $\left|\psi_{5}\right\rangle=\left|2,1, \frac{3}{2}, \frac{1}{2}\right\rangle,\left|\psi_{6}\right\rangle=\left|2,1, \frac{1}{2}, \frac{1}{2}\right\rangle,\left|\psi_{7}\right\rangle=\left|2,1, \frac{3}{2},-\frac{1}{2}\right\rangle$, and $\left|\psi_{8}\right\rangle=\left|2,1, \frac{1}{2},-\frac{1}{2}\right\rangle$, I get the block diagonal matrix $\hat{H}^{\prime}$ below

$$
\hat{H}^{\prime}=-\left[\begin{array}{cccccccc}
5 \gamma-\beta & 0 & 0 & 0 & 0 & 0 & 0 & 0 \\
0 & 5 \gamma+\beta & 0 & 0 & 0 & 0 & 0 & 0 \\
0 & 0 & \gamma-2 \beta & 0 & 0 & 0 & 0 & 0 \\
0 & 0 & 0 & \gamma+2 \beta & 0 & 0 & 0 & 0 \\
0 & 0 & 0 & 0 & \gamma-\frac{2}{3} \beta & \frac{\sqrt{2}}{3} \beta & 0 & 0 \\
0 & 0 & 0 & 0 & \frac{\sqrt{2}}{3} \beta & 5 \gamma-\frac{1}{3} \beta & 0 & 0 \\
0 & 0 & 0 & 0 & 0 & 0 & \gamma+\frac{2}{3} \beta & \frac{\sqrt{2}}{3} \beta \\
0 & 0 & 0 & 0 & 0 & 0 & \frac{\sqrt{2}}{3} \beta & 5 \gamma+\frac{1}{3} \beta
\end{array}\right]
$$


We will only need to diagonalize the $2 \times 2$ matrices

$$
-\left[\begin{array}{cc}
\gamma-\frac{2}{3} \beta & \frac{\sqrt{2}}{3} \beta \\
\frac{\sqrt{2}}{3} \beta & 5 \gamma-\frac{1}{3} \beta
\end{array}\right]
$$

and

$$
-\left[\begin{array}{cc}
\gamma+\frac{2}{3} \beta & \frac{\sqrt{2}}{3} \beta \\
\frac{\sqrt{2}}{3} \beta & 5 \gamma+\frac{1}{3} \beta
\end{array}\right]
$$

to obtain the good basis.

Explain why you agree or disagree with each student.

The QuILT strives to help students learn that student 1's approach is valid, but student 2 uses a more efficient approach that is less prone to errors in obtaining a good basis. Students are asked to summarize in words how to determine a good basis and the first-order corrections to the energy spectrum of the hydrogen atom for the Zeeman effect. Students are then asked to calculate a good basis and the first-order corrections to the energy spectrum for the $n=2$ subspace. They are provided checkpoints that allow them to reconcile any differences between their initial reasoning and the correct reasoning provided in the checkpoints.

\section{EVALUATION OF THE QUILT}

Once the researchers determined that the QuILT was successful in one-on-one implementation using a thinkaloud protocol, it was administered in graduate and upperlevel undergraduate classes. Both undergraduate and graduate students were given a pretest after traditional instruction in relevant concepts in DPT but before working through the tutorial. The pretests were not returned to the students after grading. The undergraduates worked through the tutorial in class for two days and were asked to work on the remainder of the tutorial as homework. The graduate students were given the tutorial as their only homework assignment for the week. After working through and submitting the completed tutorial, both groups were given the post-test in class. Students were given enough time in class to work through the pretest and post-test.

The pretest and post-test results for Q1 are summarized in Table I and suggest that the QuILT was helpful in reducing student difficulties with these concepts. In particular, $83 \%$ of the graduate students and $97 \%$ of the undergraduate students correctly identified that a good basis for the intermediate field Zeeman effect is option $\mathrm{v}$ in Q1. All of these students chose option v in Q1 as the only correct answer. We note that $10 \%$ of the graduate students incorrectly selected options i, ii, and iv on the post-test, while an additional $7 \%$ of the graduate students incorrectly selected both options i and ii. We also note that students were permitted to select more than one option in question
Q1, and therefore, the sum of the percentages for all of the options on the pretest and post-test is greater than $100 \%$. In addition, over $80 \%$ of the undergraduates and more than $65 \%$ of the graduate students provided correct reasoning for why they chose option $\mathrm{v}$ in Q1. For example, the following was a written response in the post-test, "Neither (coupled representation or uncoupled representation) work. We must diagonalize $\hat{H}^{\prime}$ in the degenerate subspace to find a (basis consisting of a) linear combination of states (in the coupled or uncoupled representation)." After engaging with the QuILT, the majority of the students correctly chose that neither a basis consisting of states in the coupled representation nor a basis consisting of states in the uncoupled representation form a good basis and displayed correct reasoning for their answer on the post-test.

As can be seen in Table I, the graduate students and undergraduate students generally performed at about the same level on Q1 on the pretest. However, the undergraduates outperformed the graduate students on the posttest in identifying the options in Q1 that form a good basis $(p=0.040)$. One possible explanation for the undergraduates outperforming the graduate students on the post-test could be the grade incentive associated with the QuILT. The QuILT accounted for a larger percent of the undergraduates' overall course grade and the components of the QuILT were accounted for differently for the course grade for the two groups of students. In particular, the post-test for the undergraduate students was graded for correctness in both years while the post-test for the graduate students was graded for completeness in year 1 and for correctness in year 2. Additionally, the undergraduate students knew that the material from the QuILT could appear on their examinations while the graduate students were told by the graduate instructor that this material was a review of the undergraduate quantum mechanics and that no material from the QuILT would appear on their examinations. But rather, more complex problems on the DPT would appear on the exams. The fact that the graduate students were given very small grade incentive to learn the material in the QuILT may have decreased their motivation to engage as deeply with the QuILT as the undergraduates and may explain why the graduate students did not perform as well as the undergraduate students on the post-test. Another possible reason for the differences in the post-test scores may be that undergraduates engaged in collaborative group learning while working on the QuILT for two class periods, whereas the graduate students worked on it as a homework assignment with less collaboration and support.

\section{SUMMARY}

Both upper-level undergraduate and graduate students struggled with finding perturbative corrections to the hydrogen atom energy spectrum for the intermediate field Zeeman effect using DPT. Interviewed students' responses suggested that some of them held epistemological beliefs 
inconsistent with the framework of QM and struggled with mathematical sense making in the context of QM in which the paradigm is novel [22]. After traditional instruction, some students claimed that different initial choice of the basis before a good basis has been found will yield different corrections to the energy spectrum of the hydrogen atom for the Zeeman effect. These students had difficulty in connecting experimental observations with quantum theory and in correctly reasoning that since the corrections to the energy spectrum can be measured experimentally, different choices of the initial basis cannot yield different physically observable corrections to the energy spectrum. Since students are still developing expertise in QM and the DPT requires appropriate integration of mathematical and physical concepts, cognitive overload can be high while reasoning about these problems [65]. Advanced students found it challenging to do metacognition [65] in this context of QM and provided responses that were not consistent with each other.

Using the common difficulties of advanced students with the corrections to the energy spectrum of the hydrogen atom for the intermediate field Zeeman effect, we developed and evaluated a research-based QuILT which focuses on helping students reason about and find a good basis for the Zeeman effect. Since the DPT requires students to apply advanced mathematical concepts in the context of a concrete physical problem, students often struggled to connect and apply mathematics correctly in the physics context. For example, in order to be able to determine a good basis and corrections to the energies for the Zeeman effect, one must have a strong background in linear algebra and be able to apply it in the context of solving quantum physics problem involving DPT for the intermediate field Zeeman effect. Since students' working memory while solving these problems involving the Zeeman effect is constrained to a limited number of "chunks," cognitive load may become high and it may become challenging for many students to be able to do sufficient metacognition without appropriate guidance and scaffolding support.

The QuILT strives to provide appropriate scaffolding and feedback using a guided inquiry-based approach to help students develop a functional understanding of relevant concepts. The evaluation shows that the QuILT is effective in improving students' understanding of the perturbative corrections to the energy spectrum of the hydrogen atom for the Zeeman effect. In particular, both on the written post-test and during interviews, student responses afforded opportunity to probe their reasoning. We find that the QuILT helped students reason about DPT more consistently and be able to reason about why neither a basis consisting of states in the coupled nor the uncoupled representation forms a good basis for the Zeeman effect.

\section{ACKNOWLEDGMENTS}

We thank the NSF for Grant No. PHY-1505460 and 1806691. We are also thankful to members of the Department of Physics and Astronomy at the University of Pittsburgh (especially R. P. Devaty). Additionally, we thank the students who interviewed to help improve the QuILT and our understanding of student difficulties.
[1] G. Ireson, A multivariate analysis of undergraduate physics students' conceptions of quantum phenomena, Eur. J. Phys. 20, 193 (1999).

[2] V. Dini and D. Hammer, Case study of a successful learner's epistemological framings of quantum mechanics, Phys. Rev. Phys. Educ. Res. 13, 010124 (2017).

[3] A. Johansson, Undergraduate quantum mechanics: Lost opportunities for engaging motivated students?, Eur. J. Phys. 39, 025705 (2018).

[4] F. M. Toyana and Y. Nogami, Comment on overcoming misconceptions in quantum mechanics with the time evolution operator, Eur. J. Phys. 34, L73 (2013).

[5] L. M. Arevalo Aguilar, F. Velasco Luna, C. RobledoSanchez, and M. L. Arroyo-Carrasco, The infinite square well potential and the evolution operator method for the purpose of overcoming misconceptions in quantum mechanics, Eur. J. Phys. 35, 025001 (2014).

[6] S. Sharma and P. K. Ahluwalia, Diagnosing alternative conceptions of Fermi energy among undergraduate students, Eur. J. Phys. 33, 883 (2012).
[7] E. Gire and C. Manogue, Making sense of operators, eigenstates, and quantum measurements, AIP Conf. Proc. 1413, 195 (2012).

[8] C. Singh, Student understanding of quantum mechanics, Am. J. Phys. 69, 885 (2001).

[9] A. Kohnle, I. Bozhinova, D. Browne, M. Everitt, A. Fomins, P. Kok, G. Kulaitis, M. Prokopas, D. Raine, and E. Swinbank, A new introductory quantum mechanics curriculum, Eur. J. Phys. 35, 015001 (2014).

[10] A. Kohnle, M. Douglass, T. J. Edwards, A. D. Gillies, C. A. Hooley, and B.D. Sinclair, Developing and evaluating animations for teaching quantum mechanics concepts, Eur. J. Phys. 31, 1441 (2010).

[11] D. Domert, C. Linder, and A. Ingerman, Probability as a conceptual hurdle to understanding one-dimensional quantum scattering and tunnelling, Eur. J. Phys. 26, 47 (2005).

[12] C. Singh, Student understanding of quantum mechanics at the beginning of graduate instruction, Am. J. Phys. 76, 277 (2008). 
[13] R. Muller and H. Wiesner, Teaching quantum mechanics on an introductory level, Am. J. Phys. 70, 200 (2002).

[14] D. Zollman, N. S. Rebello, and K. Hogg, Quantum mechanics for everyone: Hands-on activities integrated with technology, Am. J. Phys. 70, 252 (2002).

[15] A. Maries, R. Sayer, and C. Singh, Investigating transfer of learning in advanced quantum mechanics, in Proceedings of the Physics Education Research Conference 2015, College Park, MD, edited by A. Churukian, D. Jones, and L. Ding (2015), pp. 207-210, http://dx.doi.org/10 .1119/perc.2015.pr.047.

[16] C. Singh and E. Marshman, Analogous patterns of student reasoning difficulties in introductory physics and upperlevel quantum mechanics, in Proceedings of the 2013 Physics Education Research Conference 2013, Portland, $O R$, edited by P. Engelhardt, A. Churukian, and D. Jones (2014), pp. 46-49, http://dx.doi.org/10.1119/perc.2013.inv .010 .

[17] S. Siddiqui and C. Singh, Surveying instructors' attitudes and approaches to teaching quantum mechanics, AIP Conf. Proc. 1289, 297 (2010).

[18] G. Zhu and C. Singh, Surveying students' understanding of quantum mechanics in one spatial dimension, Am. J. Phys. 80, 252 (2012).

[19] C. Singh and G. Zhu, Surveying students' understanding of quantum mechanics, AIP Conf. Proc. 1289, 301 (2010).

[20] C. Singh and G. Zhu, Cognitive issues in learning advanced physics: An example from quantum mechanics, AIP Conf. Proc. 1179, 63 (2009).

[21] S. Y. Lin and C. Singh, Categorization of quantum mechanics problems by professors and students, Eur. J. Phys. 31, 57 (2010).

[22] E. Marshman and C. Singh, Framework for understanding the patterns of student difficulties in quantum mechanics, Phys. Rev. ST Phys. Educ. Res. 11, 020119 (2015).

[23] M. Wittmann, R. Steinberg, and E. Redish, Investigating student understanding of quantum physics: Spontaneous models of conductivity, Am. J. Phys. 70, 218 (2002).

[24] G. Passante, P. J. Emigh, and P. S. Shaffer, Examining student ideas about energy measurements on quantum states across undergraduate and graduate level, Phys. Rev. ST Phys. Educ. Res. 11, 020111 (2015).

[25] P. J. Emigh, G. Passante, and P. S. Shaffer, Student understanding of time dependence in quantum mechanics, Phys. Rev. ST Phys. Educ. Res. 11, 020112 (2015).

[26] E. Marshman and C. Singh, Student difficulties with quantum states while translating state vectors in Dirac notation to wave functions in position and momentum representations, in Proceedings of the Physics Education Research Conference 2015, College Park, MD, edited by A. Churukian, D. Jones, and L. Ding (2015), pp. 211-214, http://dx.doi.org/10.1119/perc.2015.pr.048.

[27] E. Marshman and C. Singh, Investigating student difficulties with time dependence of expectation values in quantum mechanics, in Proceedings of the Physics Education Research Conference 2013, Portland, OR, edited by P. Engelhardt, A. Churukian, and D. Jones (2014), p. 245, http://dx.doi.org/10.1119/perc.2013.pr.049.

[28] G. Zhu and C. Singh, Students' understanding of SternGerlach experiment, AIP Conf. Proc. 1179, 309 (2009).
[29] C. Singh and E. Marshman, Review of student difficulties in upper-level quantum mechanics, Phys. Rev. ST Phys. Educ. Res. 11, 020117 (2015).

[30] C. Singh, Transfer of learning in quantum mechanics, AIP Conf. Proc. 790, 23 (2005).

[31] C. Singh, Student difficulties with quantum mechanics formalism, AIP Conf. Proc. 883, 185 (2007).

[32] B. Brown, A. Mason, and C. Singh, The effect of giving explicit incentives to correct mistakes on subsequent problem solving in quantum mechanics, in Proceedings of the 2015 Physics Education Research Conference 2015, College Park, MD, edited by A. Churukian, D. Jones, and L. Ding (2015), pp. 67-70, http://dx.doi.org/10.1119/perc .2015.pr.012.

[33] C. Singh and E. Marshman, Investigating student difficulties with Dirac notation, in Proceedings of the 2013 Physics Education Research Conference 2013, Portland, $O R$, edited by P. Engelhardt, A. Churukian, and D. Jones (2014), pp. 345-348, http://dx.doi.org/10.1119/perc.2013 .pr.074.

[34] A. J. Mason and C. Singh, Do advanced students learn from their mistakes without explicit intervention?, Am. J. Phys. 78, 760 (2010).

[35] C. Singh, M. Belloni, and W. Christian, Improving students' understanding of quantum mechanics, Phys. Today 59, No. 8, 43 (2006).

[36] G. Zhu and C. Singh, Improving students' understanding of quantum mechanics via the Stern-Gerlach experiment, Am. J. Phys. 79, 499 (2011).

[37] G. Zhu and C. Singh, Improving students' understanding of quantum measurement. I. Investigation of difficulties, Phys. Rev. ST Phys. Educ. Res. 8, 010117 (2012).

[38] G. Zhu and C. Singh, Improving students' understanding of quantum measurement. II. Development of researchbased learning tools, Phys. Rev. ST Phys. Educ. Res. 8, 010118 (2012).

[39] G. Zhu and C. Singh, Improving student understanding of addition of angular momentum in quantum mechanics, Phys. Rev. ST Phys. Educ. Res. 9, 010101 (2013).

[40] C. Singh and G. Zhu, Improving students' understanding of quantum mechanics in one spatial dimension, AIP Conf. Proc. 1413, 77 (2012).

[41] C. Singh, Assessing and improving student understanding of quantum mechanics, AIP Conf. Proc. 818, 69 (2006).

[42] C. Singh, Helping students learn quantum mechanics for quantum computing, AIP Conf. Proc. 883, 42 (2007).

[43] B. Brown, A. Mason, and C. Singh, Improving performance in quantum mechanics with explicit incentives to correct mistakes, Phys. Rev. Phys. Educ. Res. 12, 010121 (2016).

[44] C. Singh, Interactive learning tutorials on quantum mechanics, Am. J. Phys. 76, 400 (2008).

[45] S. DeVore and C. Singh, Development of an interactive tutorial on quantum key distribution, in Proceedings of the 2014 Physics Education Research Conference 2014, Minneapolis, MN, edited by P. Engelhardt, A. Churukian, and D. Jones (2015), pp. 59-62, http://dx.doi.org/10.1119/perc .2014.pr.011.

[46] C. Singh and E. Marshman, Developing an interactive tutorial on a Mach-Zehnder interferometer with single 
photons, in Proceedings of the 2014 Physics Education Research Conference 2014, Minneapolis, MN, edited by P. Engelhardt, A. Churukian, and D. Jones (2015), pp. 239-242, http://dx.doi.org/10.1119/perc.2014.pr.056.

[47] E. Marshman and C. Singh, Interactive tutorial to improve student understanding of single photon experiments involving a Mach-Zehnder interferometer, Eur. J. Phys. 37, 024001 (2016).

[48] C. Singh and E. Marshman, Developing an interactive tutorial on a quantum eraser, in Proceedings of the Physics Education Research Conference 2014, Minneapolis, MN, edited by P. Engelhardt, A. Churukian, and D. Jones (2015), pp. 175-178, http://dx.doi.org/10.1119/perc.2014 .pr.040.

[49] G. Zhu and C. Singh, Improving students' understanding of quantum measurement, AIP Conf. Proc. 1289, 345 (2010).

[50] B. Brown and C. Singh, Development and evaluation of a quantum interactive learning tutorial on Larmor Precession of spin, in Proceedings of the Physics Education Research Conference 2014, Minneapolis, MN, edited by P. Engelhardt, A. Churukian, and D. Jones (2015), pp. 47-50, http://dx.doi.org/10.1119/perc.2014.pr.008.

[51] E. Marshman and C. Singh, Investigating and improving student understanding of the probability distributions for measuring physical observables in quantum mechanics, Eur. J. Phys. 38, 025705 (2017).

[52] E. Marshman and C. Singh, Investigating and improving student understanding of quantum mechanics in the context of single photon interference, Phys. Rev. Phys. Educ. Res. 13, 010117 (2017).

[53] E. Marshman and C. Singh, Investigating and improving student understanding of the expectation values of observables in quantum mechanics, Eur. J. Phys. 38, 045701 (2017).

[54] R. Sayer, A. Maries, and C. Singh, A quantum interactive learning tutorial on the double-slit experiment to improve student understanding of quantum mechanics, Phys. Rev. Phys. Educ. Res. 13, 010123 (2017).

[55] E. Marshman and C. Singh, Investigating and improving student understanding of quantum mechanical observables and their corresponding operators in Dirac notation, Eur. J. Phys. 39, 015707 (2018).

[56] A. Maries, R. Sayer, and C. Singh, Effectiveness of interactive tutorials in promoting "which-path" information reasoning in advanced quantum mechanics, Phys. Rev. Phys. Educ. Res. 13, 020115 (2017).

[57] C. Keebaugh, E. Marshman, and C. Singh, Developing and evaluating an interactive tutorial on degenerate perturbation theory, in Proceedings of the Physics Education Conference 2016, Sacramento, CA, edited by
D. Jones, L. Ding, and A. Traxler (2016), pp. 184-187, http://dx.doi.org/10.1119/perc.2016.pr.041.

[58] C. Keebaugh, E. Marshman, and C. Singh, Investigating and addressing student difficulties with the corrections to the energies of the hydrogen atom for the strong and weak field Zeeman effect, Eur. J. Phys. 39, 045701 (2018).

[59] C. Keebaugh, E. Marshman, and C. Singh, Investigating and addressing student difficulties with a good basis for finding perturbative corrections in the context of degenerate perturbation theory, Eur. J. Phys. 39, 055701 (2018).

[60] J. Tuminaro and E. Redish, Elements of a cognitive model of physics problem solving: Epistemic games, Phys. Rev. ST Phys. Educ. Res. 3, 020101 (2007).

[61] D. Hammer, A. Elby, R. E. Scherr, and E. F. Redish, Resources, framing, and transfer, in Transfer of Learning from a Modern Multidisciplinary Perspective, edited by J. Mestre (Information Age Publishing, Greenwich, 2005), pp. 89-120.

[62] A. Maries, S. Y. Lin, and C. Singh, The impact of students' epistemological framing on a task requiring representational consistency, in Proceedings of the 2016 Physics Education Research Conference 2016, Sacramento, $C A$, edited by D. Jones, L. Ding, and A. Traxler (2016), pp. 212-215, http://dx.doi.org/10.1119/perc.2016.pr.048.

[63] A. Maries, S. Y. Lin, and C. Singh, Challenges in designing appropriate scaffolding to improve students' representational consistency: The case of a Gauss's law problem, Phys. Rev. Phys. Educ. Res. 13, 020103 (2017).

[64] J. Li and C. Singh, Students' difficulties with equations involving circuit elements, AIP Conf. Proc. 1413, 243 (2012).

[65] J. Sweller, Cognitive load theory, learning difficulty, and instructional design, Learn. Instr. 4, 295 (1994).

[66] D. Griffiths, Introduction to Quantum Mechanics (Prentice Hall, Englewood Cliffs, NJ, 2005), pp. 221-252.

[67] M. T. H. Chi, in The Think Aloud Method: A Practical Guide to Modeling Cognitive Processes, edited by M. W. van Someren, Y.F. Barnard, and J. A.C. Sandberg (Academic Press, London, 1994), pp. 1-12.

[68] https://sites.google.com/site/quiltbeta/degenerateperturbation-theory-1.

[69] A Guide to Task Analysis, edited by B. Kirwan and L. K. Ainsworth (CRC Press, London, 1992).

[70] J. Piaget, Success and Understanding (Harvard University Press, Cambridge, MA, 1978).

[71] D. Schwartz, J. Bransford, and D. Sears, Efficiency and innovation in transfer, in Transfer of Learning from a Modern Multidisciplinary Perspective, edited by J. Mestre (Information Age, Greenwich, 2005), pp. 1-51. 